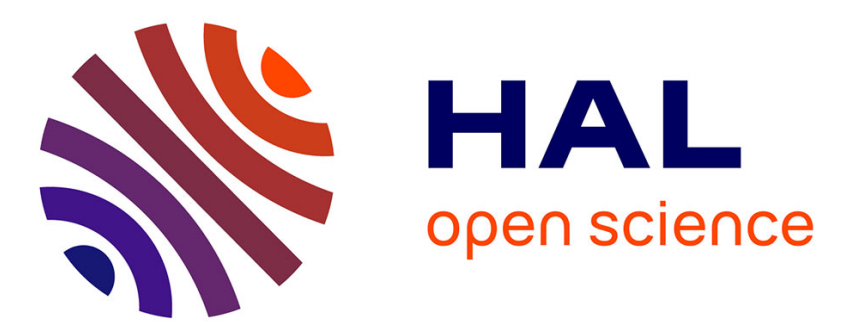

\title{
Dispersion of carbon nanotubes in polypropylene via multilayer coextrusion: Influence on the mechanical properties
}

Guillaume Miquelard-Garnier, Alain Guinault, D Fromonteil, S Delalande, Cyrille Sollogoub

\section{To cite this version:}

Guillaume Miquelard-Garnier, Alain Guinault, D Fromonteil, S Delalande, Cyrille Sollogoub. Dispersion of carbon nanotubes in polypropylene via multilayer coextrusion: Influence on the mechanical properties. Polymer, 2013, 54 (16), pp.4290-4297. 10.1016/j.polymer.2013.06.007 . hal-00994511

\section{HAL Id: hal-00994511 https://hal.science/hal-00994511}

Submitted on 21 May 2014

HAL is a multi-disciplinary open access archive for the deposit and dissemination of scientific research documents, whether they are published or not. The documents may come from teaching and research institutions in France or abroad, or from public or private research centers.
L'archive ouverte pluridisciplinaire HAL, est destinée au dépôt et à la diffusion de documents scientifiques de niveau recherche, publiés ou non, émanant des établissements d'enseignement et de recherche français ou étrangers, des laboratoires publics ou privés. 


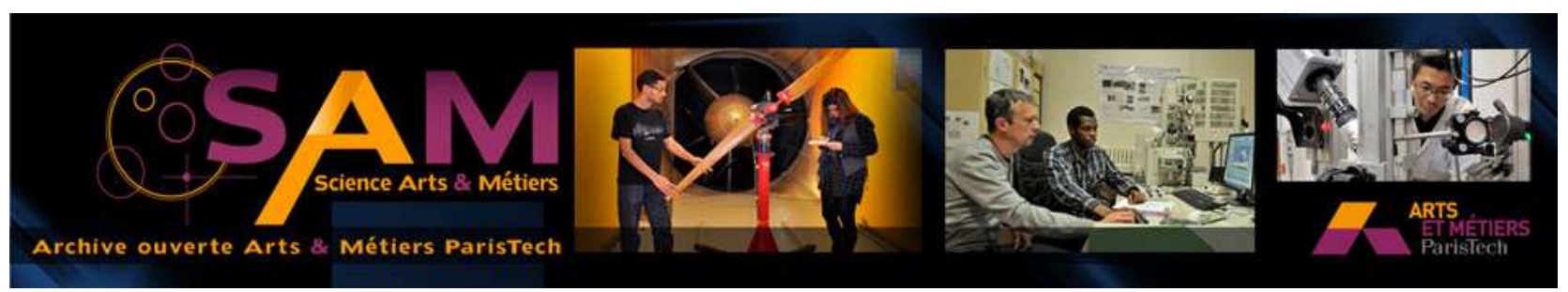

Science Arts \& Métiers (SAM)

is an open access repository that collects the work of Arts et Métiers ParisTech researchers and makes it freely available over the web where possible.

This is an author-deposited version published in: http://sam.ensam.eu Handle ID: .http://hdl.handle.net/10985/8175

\section{To cite this version :}

Guillaume MIQUELARD-GARNIER, Alain GUINAULT, D FROMONTEIL, S DELALANDE, Cyrille SOLLOGOUB - Dispersion of carbon nanotubes in polypropylene via multilayer coextrusion: Influence on the mechanical properties - Polymer - Vol. 54, n¹6, p.4290-4297 - 2013 


\title{
Dispersion of carbon nanotubes in polypropylene via multilayer coextrusion: Influence on the mechanical properties
}

\author{
G. Miquelard-Garnier ${ }^{\mathrm{a}, \mathrm{b}, *}$, A. Guinault ${ }^{\mathrm{a}, \mathrm{b}}$, D. Fromonteil ${ }^{\mathrm{c}}$, S. Delalande ${ }^{\mathrm{c}}$, C. Sollogoub ${ }^{\mathrm{a}, \mathrm{b}}$ \\ a PIMM, Arts et Métiers ParisTech, 151 boulevard de l'Hôpital, 75013 Paris, France \\ ${ }^{\mathrm{b}}$ P-2AM, CNAM, 292 rue Saint-Martin, 75003 Paris, France \\ ${ }^{c}$ PSA Peugeot Citroën, Centre Technique de Vélizy, 78140 Velizy Villacoublay, France
}

Keywords:

Carbon nanotube

Multilayer coextrusion

Nanocomposite

\begin{abstract}
A B S T R A C T
Multilayer coextrusion was used to disperse Carbon Nanotubes (CNT) in polypropylene (PP). The dilution of commercially available masterbatches using a twin-screw extruder was first applied to produce several formulations, which were then mixed with PP using a multilayer coextrusion device to obtain films or pellets with CNT concentrations between 0.1 and $1 \% \mathrm{wt}$. The influence of the specific mechanical energy (SME) during the dilution step, of the addition of a compatibilizer, and of the multilayer tool on the CNT dispersion within the matrix was highlighted. The effect of the dispersion on the thermomechanical properties of the resulting materials was studied. We showed notably that films containing $0.2 \%$ wt CNT, $1 \%$ wt of PPgAm, prepared at high SME presented a Young's modulus increase of $25-30 \%$ without significant decrease in the elongation at break. These results, using low amounts of CNT and industrially available devices, may show a new path for producing nanocomposites.
\end{abstract}

\section{Introduction}

Carbon nanotubes (CNT)/polymer composites have received a huge amount of interest over the past ten years due to the unique combination of properties of nanotubes, mainly their large aspect ratio (typically in the 100-1000 range), low density, extremely high tensile moduli and strengths, toughness and high electrical conductivity [1]. In consequence, they have been for quite a long time [2] considered as potentially ideal fillers in high performance polymer composites, with the idea that despite their high price even for industrial MultiWall CNT (MWCNT), a very low fraction of CNT compared to classical fillers could lead to higher reinforcements [3].

To achieve good mechanical properties of the composite, one however does need to achieve a good dispersion/distribution of the nanotubes [1]: because of large surface areas of contact possibly creating physical entanglements and interactions, nanotubes are often agglomerated, which can decrease the efficiency of the filler (local stress concentrations and smaller effective aspect ratio). Moreover, orientation of the nanotubes and compatibility between

\footnotetext{
* Corresponding author. PIMM, Arts et Métiers ParisTech, 151 boulevard de l'Hôpital, 75013 Paris, France.

E-mail addresses: guillaume.miquelard_garnier@cnam.fr (G. MiquelardGarnier), cyrille.sollogoub@cnam.fr (C. Sollogoub).
}

the polymer matrix and the nanotubes, due to their chemical structures, play an important role in the final properties of the composites.

Several methods have been studied to prepare CNT-based composites and thoroughly reviewed in the literature (see for example Refs. $[1,3,4])$.

Solution processing may be the most common method used at small scales to obtain such composites. Chemical processing can be achieved via in-situ polymerization or covalent functionalization of nanotubes [3]. However, melt processing is the only way to produce CNT-composites that is compatible with standard industrial processes (injection, extrusion, compression molding), due to its speed, cost and relative simplicity. An alternative route, solid-state shear pulverization appears promising but is still in its early stages [5-7].

Melt mixing actually has been less fundamentally studied than the others, and to this day gave the least interesting results, despite few exceptions [4]. This is mainly due to the fact that one is dealing with relatively high viscosities that affect the ability to disperse efficiently CNT in the matrixes, especially if the polymer and the CNT do not have favorable interactions (polyolefines). Reviews mentioned above list techniques or combinations of techniques to produce CNT nanocomposites in varieties of polymers but a "universal" and comprehensive method has yet to be proposed to achieve commercially relevant materials.

One of the most common industrial techniques for melt mixing is extrusion, and especially twin-screw extrusion in the case of CNT. However, only few recent papers use this method to create CNT 
composites. Pötschke et al. (for example references [8-11]) have published a large amount of work over the past few years in order to study extensively the role of different parameters using a twinscrew extruder in obtaining good dispersion of CNT within different polymer matrixes. In particular, these studies have pointed out the crucial importance of factors like matrix infiltration, masterbatch dilution technique, specific mechanical energy, screw configurations... In this paper, we propose the use of multilayer coextrusion to improve the dispersion and distribution of CNT in a polypropylene matrix to achieve interesting mechanical properties at low contents of CNT. Multilayer coextrusion is a quite old technique [12] that received under slightly various forms growing interest over the past years [13-15], following the pioneer work by Baer and his coworkers [16-21] and has been developed in our group recently [22]. In the context of this study, the idea is to use the multilayer coextrusion as a "mixing" tool: in this work we produce "layers" of the same polymer smaller than the typical size of the CNTs aggregates. Hence, the shear stress created by multilayer coextrusion should disperse and simultaneously orientate them in the extrusion direction and the confined structure should force breaking of the aggregates [23].

This idea has been scarcely studied to our knowledge: Jana et al. studied a similar mixing method to develop multilayer morphologies [24] and to disperse oxidized carbon nanofibers in PMMA and thermoplastic polyurethane with promising results in the viscoelastic region $[25,26]$. Very recently, Guo et al. studied the conductivity of polypropylene nanocomposites made using a similar device based on single extrusion $[27,28]$ but no mechanical measurements have been performed.

In this paper we show that this method is effective and scalable to industrial processes to reinforce a commodity plastic such as Polypropylene (PP). PP is, for example, widely used in the automobile industry and increasing its mechanical properties without making it brittle could lead to lighter cars.

We can mention to conclude some studies devoted to the reinforcing effect of CNT dispersed in polypropylene matrix by melt mixing. Lopez-Manchado et al. [29] studied isotactic PP (iPP) with single wall carbon nanotubes (SWCNT) and found that Young's modulus is increased by roughly $30 \%$ at $0.75 \%$ of CNT. The authors show an increase in Tc with increasing content of CNT, and conclude that CNT act as nucleating agents for PP crystals with no substantial changes in the crystalline structure. Similar observations were made by Leelapornpisit et al. [30]: the mechanical reinforcement would then be due principally to the ordered zone rather than the CNT, which could explain the somewhat "limited" effect. More recent papers looked at promising routes toward industrial processes [5-7] or combined melt processing techniques with chemical engineering, especially surface modifications of the CNT [31-33], or commented on the effect of adding a compatibilizing agent between the PP and the CNT such as maleic anhydride grafted polyolefine [34-36].

The goal of this study is to use only industrially relevant apparatus (twin-screw and multilayer coextrusion), to identify the relevant mechanical and compositional parameters and methods leading to effective nanocomposites for the automobile industry, eg cost effective (small amount of CNT), with improved thermomechanical properties.

\section{Materials and methods}

\subsection{Materials}

Polypropylene PPH5060 is a homopolymer polypropylene grade developed by Total Petrochemicals suitable for extrusion, and will be used as the matrix in the nanocomposites produced. The Melt Flow Index is $6 \mathrm{~g} / 10 \mathrm{~min}\left(230^{\circ} \mathrm{C} / 2.16 \mathrm{~kg}\right)$.
Polypropylene grafted with maleic anhydride (PPgAM) (see Fig. 1) was used in small quantity (between 0.5 and $7 \%$ wt) to increase the compatibility between the matrix and the CNT ("coupling agent"). Noncommercial Orevac PPgAM similar to the CA100 (high content of maleic anhydride) was obtained via Arkema. Due to defects occurring during their fabrication, MWCNT present polar groups such as hydroxyl groups at their surface. It has already been shown that polymers grafted with anhydride maleic improves the interactions between matrix and CNT fillers because of the polarity of the anhydride groups [34,37]. However, the exact mechanism and its consequences on the macroscopic properties are not yet fully understood since PPgAM will also affect the crystallization of the matrix [34].

CNTs were obtained in masterbatch form from Nanocyl. Plasticyl PP2001 is a concentrate of MultiWall Carbon Nanotubes (MWCNT) dispersed at 20\%wt in a polypropylene matrix (PP2001, reference from Nanocyl) suitable for extrusion process. According to Nanocyl datasheets, their industrial grade MWCNT produced via catalytic carbon vapor deposition process (CVD) have a mean diameter of $9.5 \mathrm{~nm}$, length about $1.5 \mu \mathrm{m}$ and the carbon purity is $90 \%$ while the CNT contain $10 \%$ metal oxide impurities.

All products were used as received (under pellet forms).

\subsection{Methods: sample preparation}

\subsubsection{Twin-screw extrusion}

The twin screw extruder used in this study was a Thermo Haake PTW 16-40D. The goal with twin-screw extrusion is here to dilute the CNT concentration from $20 \%$ wt to a lower concentration by diluting the masterbatch with PPH5060. This first step is necessary since the masterbatch cannot be extruded as is using the multilayer coextrusion process, due to its high viscosity.

Following the work of Pötschke and coworkers [8-11], the influence of the specific mechanical energy (SME) in the dispersion of the CNT was studied. SME is defined by

$\mathrm{SME}=\frac{\tau N}{\dot{m}}$

where $\tau$ is the torque of the screw, $N$ the speed of the screw and $\dot{m}$ the throughput. The absolute value will indeed depend from the extruder used, especially the extrusion temperature and the ratio $\mathrm{L} /$ $\mathrm{D}$ of the screw and its profile. SME is given in $\mathrm{kJ} / \mathrm{kg}$ and basically defines a "good mixing": the faster and the longer, the better.

The SME cannot be easily fixed before the experiment, since all the parameters are related and can vary slowly during the experiment. Work by Pötschke also show that the dispersion increases with increasing SME values but reaches a plateau.

As a consequence, the SME was calculated a posteriori, and we defined roughly 3 SME regimes: low SME for values below $500 \mathrm{~kJ} /$ $\mathrm{kg}$, medium SME for values between 500 and 2500, and high SME for values higher than 2500 .

In this work, the twin-screw extrusion temperature is fixed at $240{ }^{\circ} \mathrm{C}$. The varying parameters in the different formulations a.

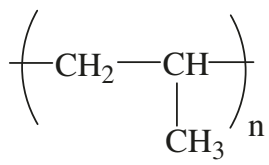

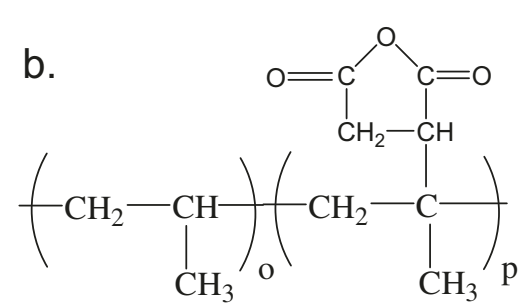

Fig. 1. a. Polypropylene. b. Polypropylene grafted with maleic anhydride PPgAM. 
produced are then the SME, the amount of compatibilizer PPgAM, and the amount of masterbatch.

The formulations produced were cooled in a water bath at the end of the extruder, and cut into pellets later to be used in the multilayer device using a mechanical grinder. The pellets obtained are later used in the lateral extruder during the multilayer coextrusion. These formulations were made with CNT concentrations from 2 to $7.5 \%$ wt with most of them at 5\%wt, which then allows producing films with final concentrations between 0.1 and $1 \% \mathrm{wt}$ after multilayer coextrusion.

\subsubsection{Multilayer coextrusion}

Using multiplying elements at the end of a classical coextrusion setup, one can multiply alternated layers of two polymers or blends while keeping the thickness of the extruded film constant, up to several thousands of layers. Films were produced with a process that combined two polymers in a classical 3-layer coextrusion feedblock (ABA). PP was extruded to form the outer skin layers and twin-screw formulations the core layer. The 3-layer polymer flow subsequently enters a mixing section, composed of a sequence of layermultiplying devices: the melt is initially cut in half vertically, and then each half is compressed and re-stretched to its original width, doubling the number of layers with each static mixer. In most cases, 10 static mixers have been used and the number of the total layers in the final sample after the mixing section should be 2049 (see Fig. 2 below). Some tests were made with 5 mixers ( 65 theoretical layers) to optically control the microstructure obtained. Finally, after passing through the last static mixer, the multilayer structure was formed into a thin sheet by passing through a flat die, $100 \mathrm{~mm}$ wide and $1 \mathrm{~mm}$ thick. The resultant films had an average thickness between 0.4 and $0.8 \mathrm{~mm}$ and width between 50 and $70 \mathrm{~mm}$.

Two laboratory single screw extruders have been used: a $30 \mathrm{~mm}$ diameter (Mapre) for the main flow and a lateral $20 \mathrm{~mm}$ diameter (Scamex) for the minor flow. The end temperatures for both extruders were fixed at $240{ }^{\circ} \mathrm{C}$.
The throughput of each extruder was adjusted to produce samples with varying final amounts of CNT. The throughput was controlled by fixing the screw speed. It is worth noting that, due to the capacities of each extruder, the typical weight ratio between the Mapre and the Scamex is between 95/5 and 80/20.

In this experiment, the theoretical number of layers $N_{\text {lay }}$ was determined by the number of multiplying elements (Fig. 2) and given by Equation (1):

$N_{\text {lay }}=1+2^{N+1}$

where $N$ is the number of multiplying elements.

Complementary injection experiments were made using a JSW J55ELII molding machine.

Strings were extruded instead of films according to the multilayer coextrusion method detailed above and pelletized. The pellets were then injection molded at $240^{\circ} \mathrm{C}$ at 800 bar during $35 \mathrm{~s}$, with $40^{\circ} \mathrm{C}$ for the mold temperature in order to obtain normalized test specimens (dog bone shaped samples following the ENISO527-2 standard).

\subsection{Methods: characterization}

\subsubsection{Optical microscopy}

Transmission optical microscopy was performed using an Olympus BH2-UMA with a 10 or $20 \times$ objective. The microscope was equipped with a camera allowing capturing pictures that can then be treated using Image J analysis software, an open source image processing program developed at the National Institutes of Health.

Samples were prepared using a Leica RM 2225 microtome. Before placing them in the microtome, the films were immerged in liquid nitrogen for $5 \mathrm{~min}$ to prevent from plasticity effects of the PP due to the cutting. The thickness of the sample was fixed at $5 \mu \mathrm{m}$ cut perpendicular to the extrusion direction throughout the film thickness.
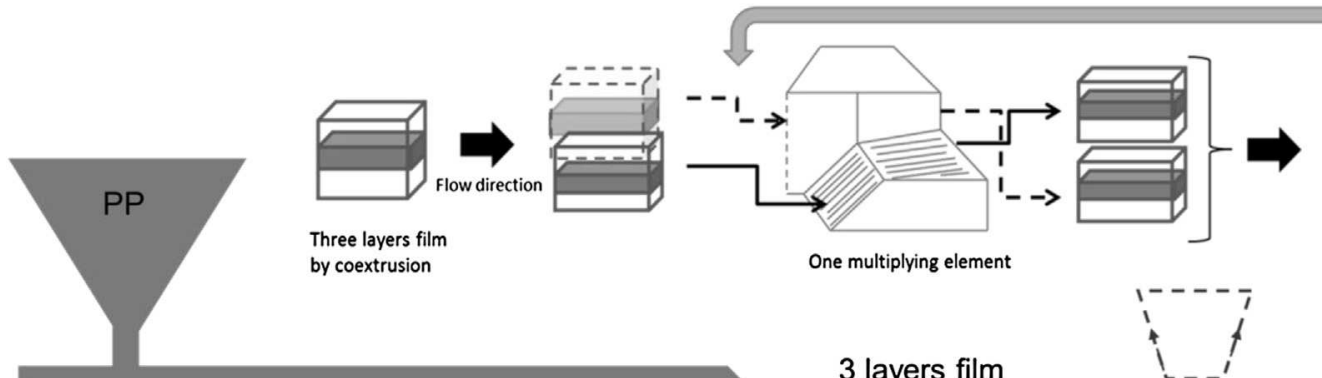

Three layers film
by coextrusion

One multiplying element

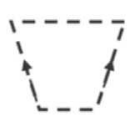

3 layers film

To multiplying elements
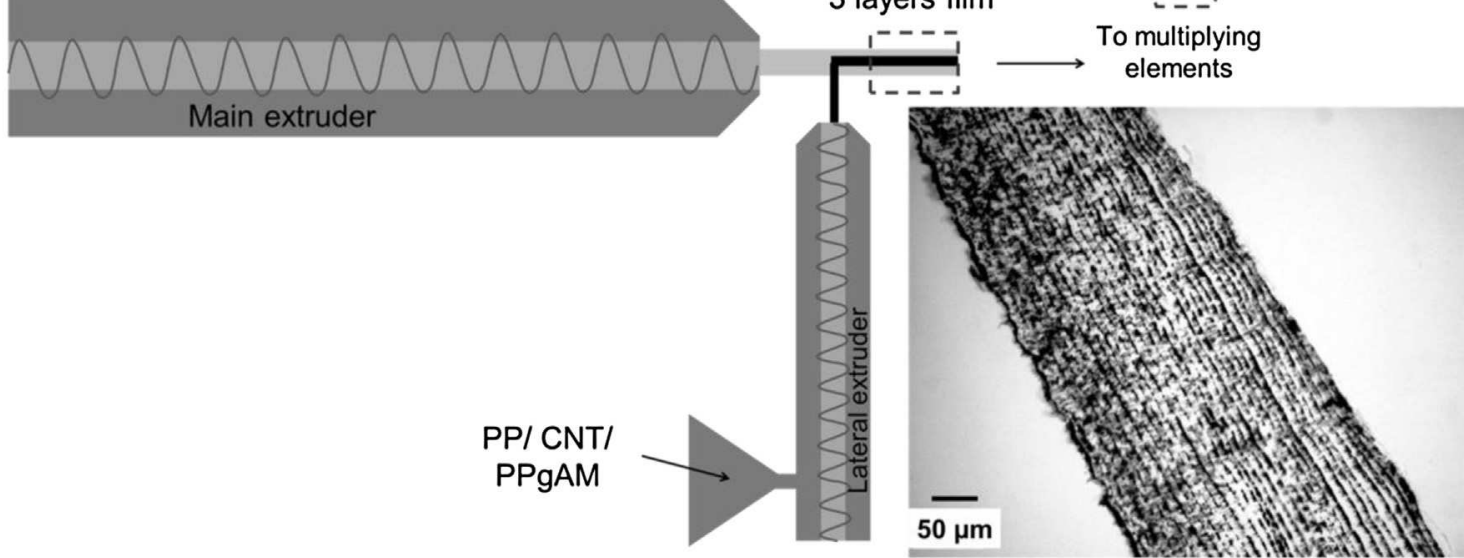

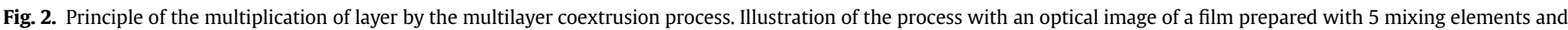
65 theoretical layers of alternating PP (white in the picture) and PP/CNT (black) (bottom right). 


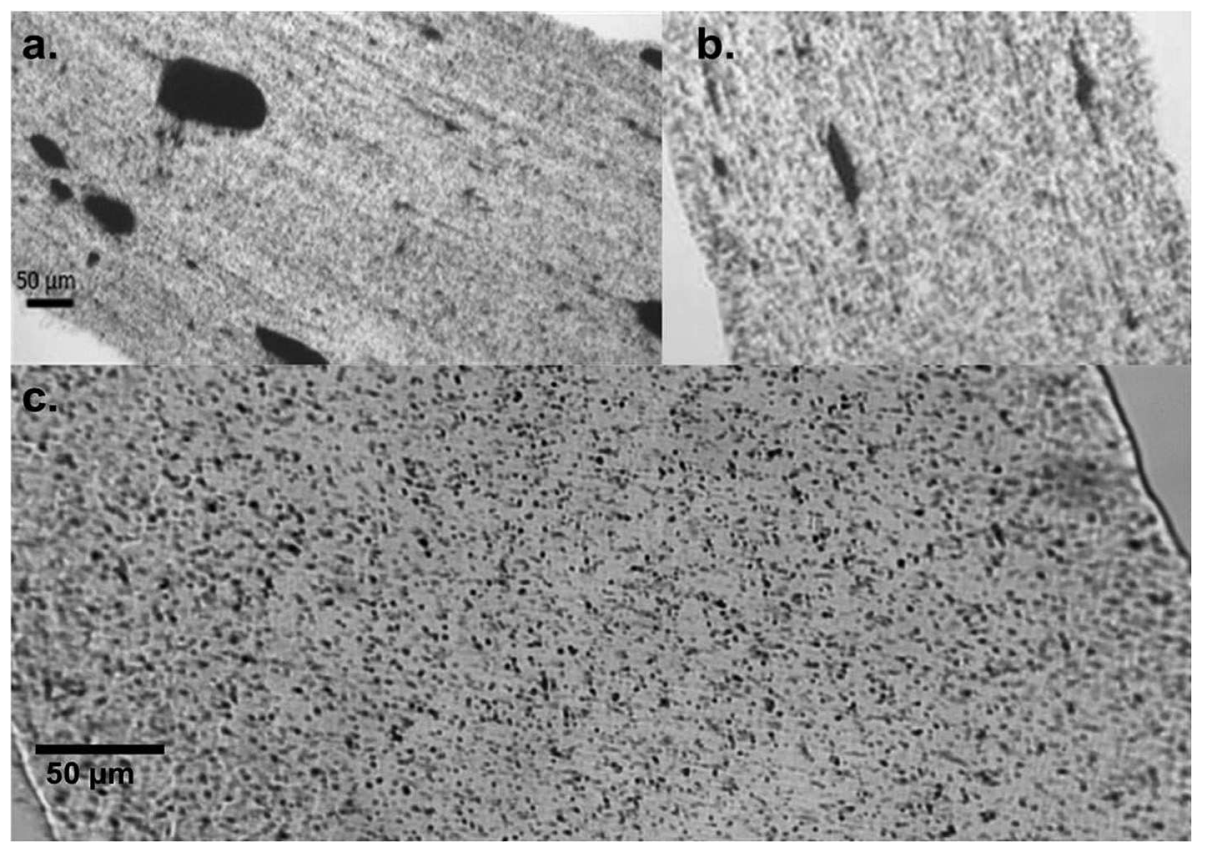

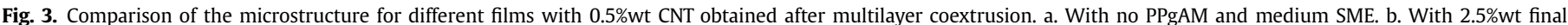
PPgAM and a medium SME used for the first dilution (a and b: same scale bar). c. With 2.5\%wt final PPgAM and a high SME.

\subsubsection{SEM}

The film cross section was observed with a HITACHI 4800 Scanning Electron Microscopy. The films were cryofractured in liquid nitrogen to obtain brittle fracture (no plastic deformation at the surface) and placed directly without further surface treatment in the SEM chamber.

\subsection{3. $D S C$}

A DSC apparatus from Perkin Elmer (Pyris 1) was used to determine the thermal transitions and the degree of crystallinity of the composite films. The DSC was calibrated with indium as a reference and the tests were performed under nitrogen flow. Cuts from the films were placed in an aluminum crucible of $40 \mu \mathrm{l}$. If necessary, several portions of films were superposed in the pan to reach a sample weight close to $10 \mathrm{mg}$. In order to obtain the crystallinity of as-prepared films, the samples were heated from $0{ }^{\circ} \mathrm{C}$ to $200{ }^{\circ} \mathrm{C}$ at $10^{\circ} \mathrm{C} / \mathrm{min}$. The degree of crystallinity of as-prepared films was obtained through the area of the melting peak. PP without CNT was also extruded trough the mixing elements under the same conditions to serve as a reference.

\subsubsection{Dynamic Mechanical Thermal Analysis (DMTA)}

DMTA was carried out on small bar samples (10.25 mm length, $5.00 \mathrm{~mm}$ width, and a thickness between 0.4 and $0.8 \mathrm{~mm}$ ) using a Triton Tritec apparatus, working in dynamic tensile mode. The frequency was set at $1 \mathrm{~Hz}$ and the dynamic displacement at $5 \mathrm{mi}-$ crons $(0.049 \%)$. The samples were heated from 25 to $180{ }^{\circ} \mathrm{C}$ at a heating rate of $2{ }^{\circ} \mathrm{C} \mathrm{min}^{-1}$. At least two samples were tested to average the results obtained.

\subsubsection{Uniaxial tensile tests}

Dog bone shaped samples were cut from the films $(10 \mathrm{~mm}$ in width, $115 \mathrm{~mm}$ in length) and were tested on an INSTRON 4507 machine equipped with a $5 \mathrm{kN}$ cell force, measuring force over displacement for each sample at $5 \mathrm{~mm} \mathrm{~min}^{-1} 5$ samples at least were tested for each film and the stress versus strain curves obtained from the raw data. The average values for strain at break and Young's modulus were taken. Young's modulus $E$ was calculated manually within the linear regime. Standard deviation for $E$ was found between 0.02 and 0.05 GPa for every set of samples tested, unless otherwise specified. For some samples, the failure could not be attained due to the limit of the machine and so the value of the elongation at break will be given as $>x \%$. The injected samples were tested on the same machine to measure the mechanical properties.

\section{Results}

As a first step, the industrial masterbatch needs to be diluted using twin screw extrusion before the resulting formulation can be used for the multilayer coextrusion to obtain the final films.

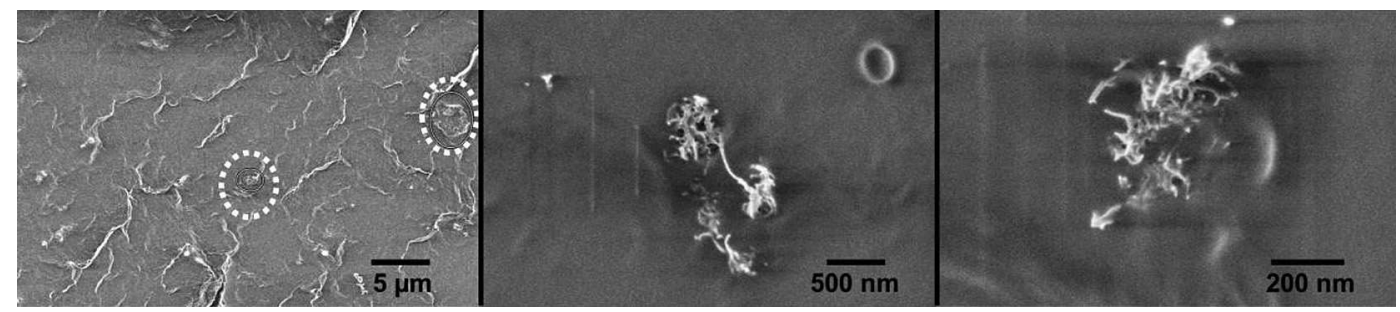

Fig. 4. SEM images of the CNT/PP nanocomposites with optimized formulations. Typical aggregates with areas around $10 \mu \mathrm{m}^{2}\left(\right.$ left), $1 \mu \mathrm{m}^{2}$ (middle), and $0.1 \mu \mathrm{m}^{2}$ (right). 
Table 1

Summary of $R$ calculated for different samples ( \pm denotes the standard deviation).

\begin{tabular}{|c|c|c|c|c|c|c|c|}
\hline & \multicolumn{2}{|c|}{ Medium SME } & \multicolumn{2}{|c|}{ Medium SME } & \multirow{2}{*}{$\begin{array}{l}\text { High SME } \\
5\end{array}$} & \multirow{2}{*}{$\frac{\text { High SME }}{23.5}$} & \multirow{2}{*}{$\begin{array}{l}\text { Injection high SME } \\
5\end{array}$} \\
\hline$P$ & 0 & & 5 & & & & \\
\hline \%wt PPgAM & 0 & 0 & 1 & 4 & 1 & 3.75 & 1 \\
\hline \%wt CNT & 0.45 & 0.9 & 0.2 & 0.8 & 0.2 & 0.16 & 0.2 \\
\hline$R(\%)$ & $4 \pm 0.5$ & $5.7 \pm 2.9$ & $2.3 \pm 1$ & $1.5 \pm 0.6$ & $0.13 \pm 0.05$ & $1.1 \pm 0.3$ & $0.06 \pm 0.02$ \\
\hline
\end{tabular}

The importance of both diluting the masterbatch with a high SME and with a blend of PPgAM and PP (contrary to reference [30] where comparisons between dilutions with pure and different PP or PPgAM were made) before using the obtained formulation in the lateral extruder is clearly illustrated in Fig. 3.

In particular, presence of PPgAM is necessary to get rid of very big aggregates (with areas which can be on the order of $1000 \mu \mathrm{m}^{2}$ ) using the multilayer coextrusion process even at low concentrations of CNT (below 1\%wt) and with 10 mixing elements (see Fig. 3a). However, working with a high SME also helps to prevent from the presence of thinner aggregates that could remain in the composite. Similar effects on the dispersion of CNT in PE matrixes were observed very recently by Pötschke et al. [38] using PEG as a compatibilizer.

However, it is important to note that adding compatibilizer results in competitive mechanisms: too low concentrations do not impact the dispersion but too high concentrations result in the reorganization of layers during the multilayer process leading to reaggregation of CNT (picture not shown). It also acts as a plasticizer which affects the mechanical properties of the matrix as will be discussed below.

Concerning the SME, we observed as Pötschke et al. [8-11] that increasing the SME favors the dispersion, or more precisely diminish the number of "big" aggregates (eg with diameters $>5 \mu \mathrm{m}$ ) in the polymer matrix (see Fig. $3 \mathrm{~b}$ and $\mathrm{c}$ ).

It is possible to quantify the dispersion using image analysis with the Image J software. Following the work of Pötschke (see most references from the bibliography section) and according to the ISO-18553 standard, the ratio $R$ between the total agglomerates with circle equivalent diameters $>5 \mu \mathrm{m}$ (aggregate area $\left.>19.6 \mu \mathrm{m}^{2}\right)$ over the total area of the sample studied was determined. For quantification at least 5 areas between 15,000 and $200,000 \mu \mathrm{m}^{2}$ were studied for every sample. The values calculated using this method however cannot be connected to the real total amount of
CNT in the sample because of the thicknesses of the samples imaged. Nevertheless the relatively high amount of aggregates measured compared to the low amount of CNT really incorporated in the sample might suggest that CNT in these "optical aggregates" are, to some extent, dispersed (but poorly) within the matrix (see Fig. 4).

It appears in Table 1 below that working at medium SME with no compatibilizer gives an average $R$ of about $5 \%$ with the presence of big aggregates (on the order of $1000 \mu \mathrm{m}^{2}$ ). Adding a 5 to 1 ratio of PPgAM compared to CNT (noted $P$ in the following) still at medium SME, leads to a reduction of $R$ for comparable concentrations of CNT $(0.9 \% \mathrm{wt}$ and $0.8 \% \mathrm{wt}$ ) from roughly 6 to $2 \%$. At this SME value, $R$ is however similar for the two concentrations of CNT studied. Increasing the SME to values above $2500 \mathrm{~kJ} / \mathrm{kg}$ leads to a further dramatic decrease of $R$, to an average value of $0.13 \%$ for a composite with $0.2 \%$ wt CNT (with almost no aggregates with areas bigger than $100 \mu \mathrm{m}^{2}$ ). It is worth noting that even at high SME, working at $P \approx 23.5$ gives an increased $R$ of $1.1 \%$ which suggests an optimum in the amount of PPgAM relative to the amount of CNT in the film. The results about the injected samples are even better but will be discussed below with more details.

These results suggest that an optimized formulation can be achieved: prepared at high SME, with concentrations below $0.5 \% \mathrm{wt}$ CNT in the film and a 5 to 1 ratio of PPgAM (for example $1 \%$ of PPgAM for $0.2 \%$ wt of CNT).

Typical aggregates from such formulations can be seen in Fig. 4 as observed by SEM. On the left, the aggregates are roughly circular with diameter slightly below $5 \mu \mathrm{m}$. On the middle and right images, smaller aggregates with sizes about 500 and $200 \mathrm{~nm}$ are showed.

The impact of big aggregates on the mechanical properties of the nanocomposites can clearly be evidenced by simple uniaxial traction experiments. The presence of big aggregates (hence high values or $R$ ) in the composite leads to a complete loss of the elongation at break of the material, from more than $400 \%$ for all samples to as low as 1 or $2 \%$ (eg before necking) with very large differences
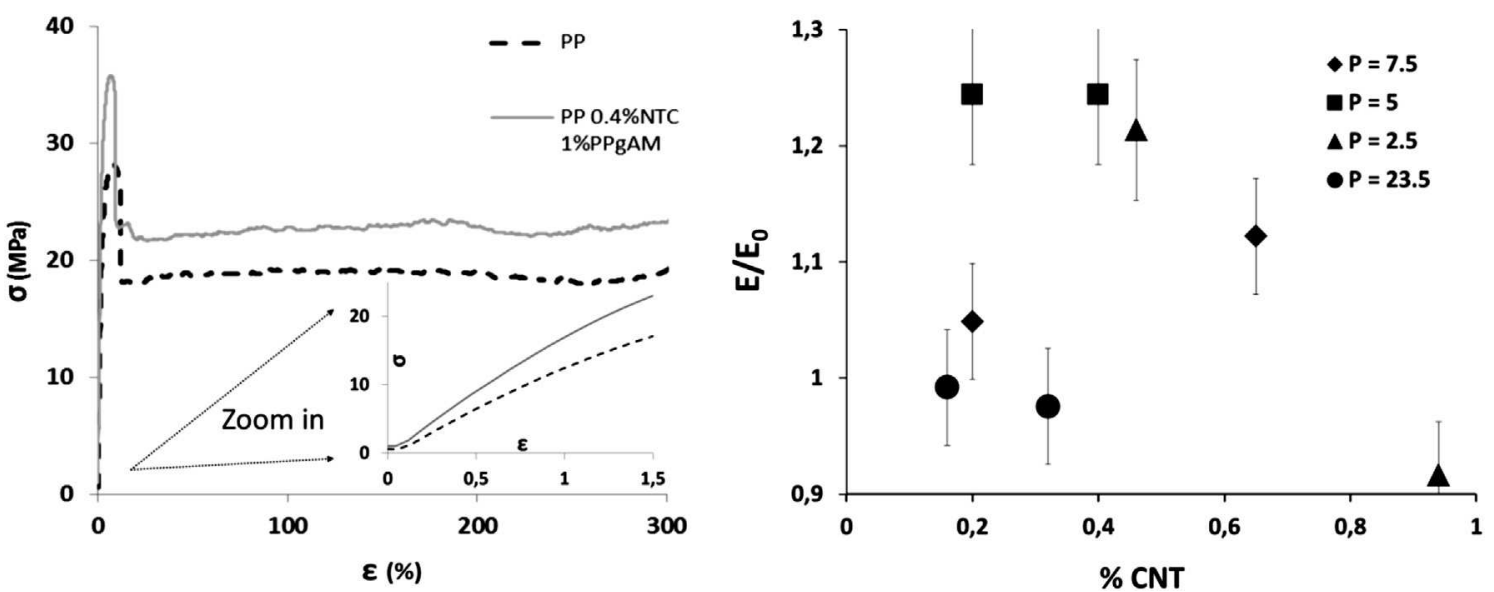

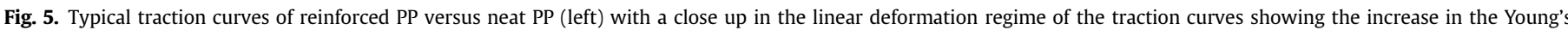

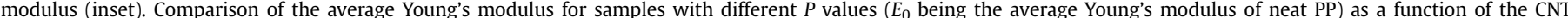

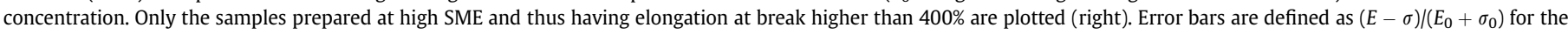
lower end and $(E+\sigma) /\left(E_{0}-\sigma_{0}\right)$ for the upper end, $\sigma$ and $\sigma_{0}$ being the standard deviation for $E$ and $E_{0}$ respectively. 


\begin{tabular}{|l|c|c|c|}
\hline \multirow{2}{*}{$\mathrm{T}\left({ }^{\circ} \mathrm{C}\right)$} & \multicolumn{3}{|c|}{$\mathrm{E}^{\prime} / \mathrm{E}^{\prime}$} \\
\cline { 2 - 4 } & $\begin{array}{l}\text { PP 0.2\% CNT } \\
1 \% \text { PPgAM }\end{array}$ & $\begin{array}{l}\text { PP 0.4\% CNT } \\
2 \% \text { PPgAM }\end{array}$ & $\begin{array}{l}\text { PP 0.16\% CNT } \\
3.75 \% \text { PPgAM }\end{array}$ \\
\hline 30 & 1.4 & 1.13 & 0.88 \\
\hline 80 & 1.58 & 1.25 & 0.96 \\
\hline
\end{tabular}

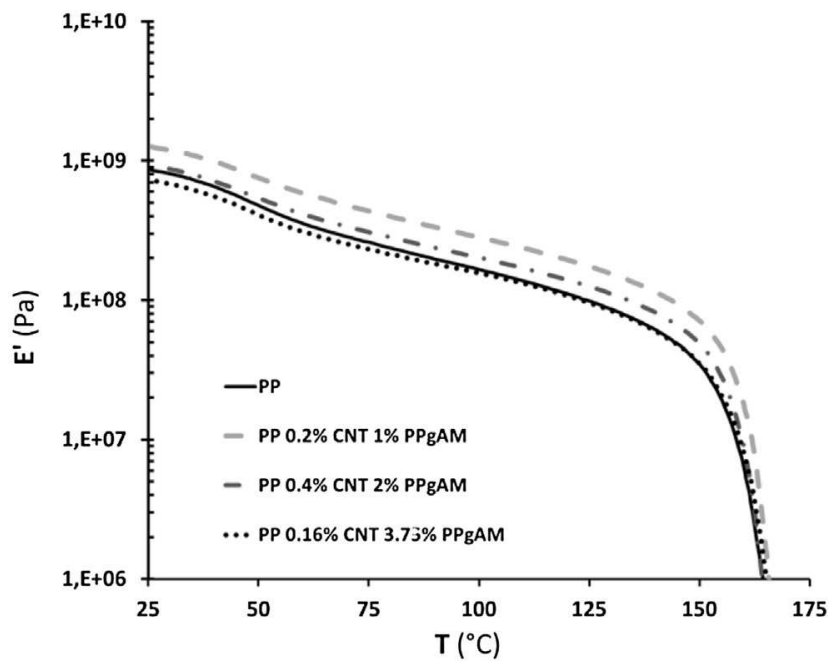

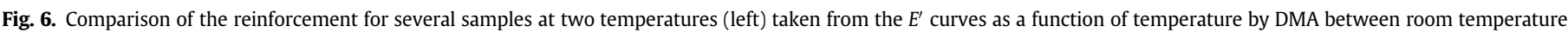
and $180^{\circ} \mathrm{C}$ (right).

between samples. However it can be noted that the modulus may increase even with such microstructures. But in that case the increase of modulus, even if reasonably high (20-30\%), does not give rise to a potentially interesting material for industrial applications since PP loses all its ability to deform.

To obtain materials with preserved elongation at break, twinscrew dilution of masterbatches must be done at high SME and with the use of well-chosen amounts of compatibilizer such as PPgAM. Interestingly, all samples made using high SME/any amount of PPgAM between 0.5 and $7 \%$ displayed elongations at break higher than $400 \%$, whereas it was not the case for any samples made at low or medium SME values. This suggests that $R$ is closely related to the resulting elongation at break of the final materials, with a threshold that might be estimated between 1 and $1.5 \%$. In consequence, we will only discuss in the following films made using formulations prepared at SEM $>2500 \mathrm{~kJ} / \mathrm{kg}$ and some amount of compatibilizer. We will however discuss below the effect of these two parameters on the reinforcement (Young's modulus), assuming that the elongation at break is preserved.

Fig. 5 shows typical curves for neat PP films and PP/CNT films prepared using the multilayer coextrusion process.

The first important point we need to assess was the effect of the process on neat PP. It was observed that the multilayer coextrusion with 10 mixing elements does not significantly affect the mechanical properties of the resulting film. Comparing a PP film prepared via simple single screw extrusion and a PP film that was prepared via multilayer coextrusion, using the same batch, leads to similar results in terms of moduli (less than $1 \%$ difference) and no significant changes in terms of elongation at break. However, different PPH5060 batches can lead to some dispersion in the data (the average modulus for the neat PP films was found to be 1.31 with a standard deviation of $0.1 \mathrm{GPa}$ ). In consequence, in the following analysis, neat PP films were always prepared for comparison with the nanocomposite films made using the same PP batches.

In the results presented in Fig. 5 (left), the lateral extruder was fed with a formulation made using $25 \%$ masterbatch (eg 5\%wt CNT), $12.5 \%$ wt PPgAM and 62.5\%wt PPH5060 with an SME around $5000 \mathrm{~kJ} /$ $\mathrm{kg}$ (and the main extruder with PPH5060). The film was produced using a 92/8 ratio between the two extruders, which gives final concentrations of $0.4 \% \mathrm{wt}$ and $1 \% \mathrm{wt}$ of CNT and PPgAM respectively. It shows that both moduli (and yield strength) increase by more than $20 \%$, while the elongation at break remains higher than $300 \%$ for the nanocomposite PP film (actually, no breaking was observed below $450 \%$-the limit of the apparatus-for all samples tested).

It appears interesting to study the increase in the average Young's modulus for every samples made at high SME, as a function of the final percentage of CNT in the material, and as a function of the ratio between \% of CNT and \% of PPgAM. Indeed, if a film prepared via multilayer coextrusion without CNT and $1 \%$ of PPgAM does not show a significant difference in terms of Young's modulus, a film containing $\sim 2.5 \%$ of PPgAM leads to a decrease of roughly $20 \%$ in the Young's modulus (around 1.1 GPa). PPgAM is acting not only as a compatibilizer, but also as a "softening agent", which suggests the good balance has to be found.

This clearly appears in the results summarized Fig. 5 (right). It shows that too little compatibilizer does not lead to a good reinforcement, while too much leads to a competition between the capacity to disperse effectively more CNT and the lowering of the mechanical properties of the matrix. In this study, the optimum film was found to be with final concentrations of $0.2 \% \mathrm{wt}$ CNT and $1 \% \mathrm{wt}$ PPgAM, with 25\% increase in the modulus while the elongation at break was fully maintained. At the same ratio between CNT and PPgAM, same reinforcement was observed with $0.4 \%$ wt CNT (hence

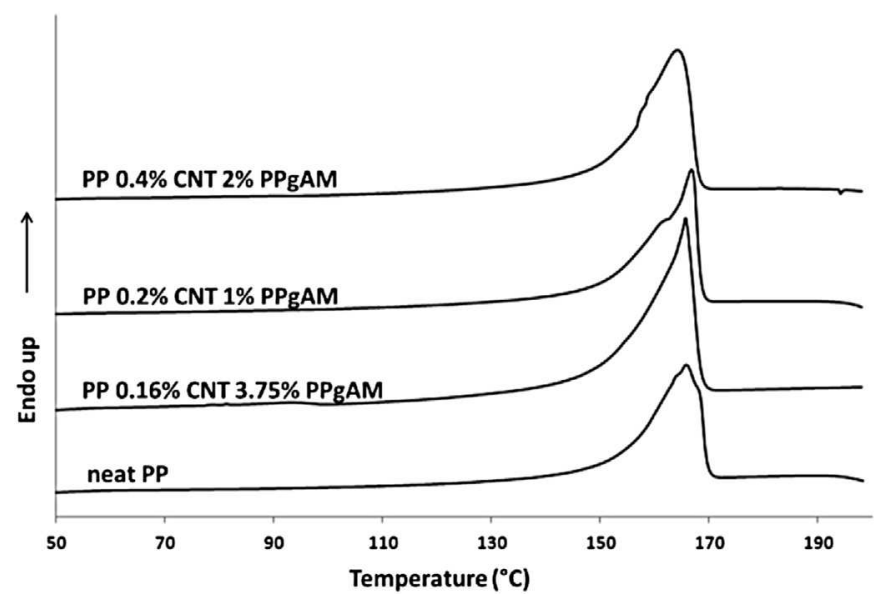

Fig. 7. DSC thermograms for different representative samples. 
Table 2

Summary of the thermal parameters obtained by DSC for representative samples.

\begin{tabular}{llll}
\hline PP & $T_{m}\left({ }^{\circ} \mathrm{C}\right)$ & $\Delta H_{m}(\mathrm{~J} / \mathrm{g})$ & $X_{c}(\%)$ \\
\hline Neat PP & $166 \pm 1$ & $92.8 \pm 2.0$ & 49 \\
PP 0.2\% CNT 1\% PPgAM & $168 \pm 1$ & $93.5 \pm 2.0$ & 49 \\
PP 0.4\% CNT 2\% PPgAM & $164 \pm 1$ & $93.3 \pm 2.0$ & 49 \\
PP 0.16\% CNT 3.75\% PPgAM & $166 \pm 1$ & $86.4 \pm 2.0$ & 45 \\
\hline
\end{tabular}

$2 \%$ of PPgAM), and similar values ( $>20 \%$ ) were obtained using $0.4 \%$ CNT and only $1 \%$ of PPgAM. However, the reinforcement decreases drastically with CNT concentrations above $0.5 \%$ with higher or lower amounts of PPgAM.

In consequence, it confirms the optical observations: the ratio $P$ has to be well chosen. Moreover this method may not be helpful to disperse high quantities of CNT $(>0.5 \% \mathrm{wt})$. However it gives remarkable results with very low amounts of $\mathrm{CNT}$ and can be useful in terms of cost effectiveness.

In comparison, a sample prepared by dry blending all ingredients at the desired concentrations (0.2\%wt CNT, $1 \%$ wt PPgAM, $98.8 \% \mathrm{wt} \mathrm{PP}$ ) in a single screw extruder leads to only $5 \%$ increase in the Young's modulus while the elongation at break goes down to $70 \%$ with large standard deviations (breaking of samples occur between $1.6 \%$ and $210 \%$ deformation).

DMTA results are presented in Fig. 6. It appears the storage modulus $E^{\prime}$ shows an increase when CNT are added using optimized formulations, whereas adding to much compatibilizer with respect to the nanotube concentration leads to a decrease of $E^{\prime}$ compared to the one of neat PP. It is difficult to compare quantitatively tensile measurements with DMTA measurements especially because of the different frequencies used and the fact that the modulus in DMTA is measured over 4 decades (or because of the compliance of the apparatus). Qualitatively however, similar trends can be observed with reinforcements of storage moduli at $30{ }^{\circ} \mathrm{C}$ of 40 and $13 \%$ respectively for PP with $0.2 \%$ wt CNT and $1 \%$ wt PPgAM, and $0.4 \%$ wt CNT and 2\%wt PPgAM respectively (25\% increase was found for both samples in tensile experiments at room temperature). More important is the fact that this reinforcement is maintained and even increased over the whole temperature range before melting. For example, at $80{ }^{\circ} \mathrm{C}$, this increase in the storage modulus is $58 \%$ and $25 \%$ respectively (left table in Fig. 6). This suggests a better ability for these materials to be used at high temperature. However, no significant differences are observed for the $\tan \delta$ peak at $155^{\circ} \mathrm{C}$ (data not shown).

Fig. 7 shows the DSC heating scans of the as-prepared composite films measured at a heating rate of $10{ }^{\circ} \mathrm{C} / \mathrm{min}$. The thermal parameters-melting temperature $\left(T_{m}\right)$, heat of fusion, degree of crystallinity $\left(X_{C}\right)$-determined from the DSC curves are summarized in Table 2. The degree of crystallinity of our samples was determined using the following relation:

$X c(\%)=\frac{\Delta H_{m}}{\Delta H_{m}^{0}} \times 100$

An enthalpy value of $190 \mathrm{~J} / \mathrm{g}$ was taken for the $100 \%$ crystalline PP homopolymer [39].

It appears from the data that the effect of CNT addition in PP matrix on $T_{m}$ and $X_{c}$ is rather marginal and indicates no clear tendency. These results seem to indicate that the incorporation of CNT in PP matrix does not affect significantly the crystallinity degree of PP, which is in agreement with other results reported in the literature for this matrix [29]. It means in particular, that the improvement of the nanocomposite mechanical properties cannot be attributed to a significant change in the matrix crystallinity degree.

The final interesting result, especially in terms of industrial application, is the possibility to apply this method to make injected pellets. We used the multilayer coextrusion with the same experimental conditions and the same formulations to produce strings (0.2\%wt CNT, $1 \%$ wt PPgAM) instead of films, which were then pelletized. The resulting pellets were then injected to produce dog bone shaped samples (see Methods). For these samples, we showed that the average modulus as measured by uniaxial tensile test increased from $1.13 \mathrm{GPa} \pm 0.14$ for the PPH5060 to $1.45 \mathrm{GPa} \pm 0.02$ (the low value for the PPH5060 modulus is probably due to the fact that it is an extrusion grade used in injection), which is a $28 \%$ increase while the elongation at break remained higher than $400 \%$ (see Fig. 8). Moreover, the aggregation ratio $R$ as defined before drops to an average of $0.06 \%$ and was below $0.1 \%$ for every picture studied with no aggregates bigger than $75 \mu \mathrm{m}^{2}$. This suggests that in that particular case, the multilayer coextrusion process is actually a dispersion tool and that no reaggregation of CNT occurs if a second processing step takes place. On the contrary, the injection step appears to improve further the dispersion.

One can conclude by comparing the results obtained to simple estimates using the Halpin-Tsai model for short fiber reinforced
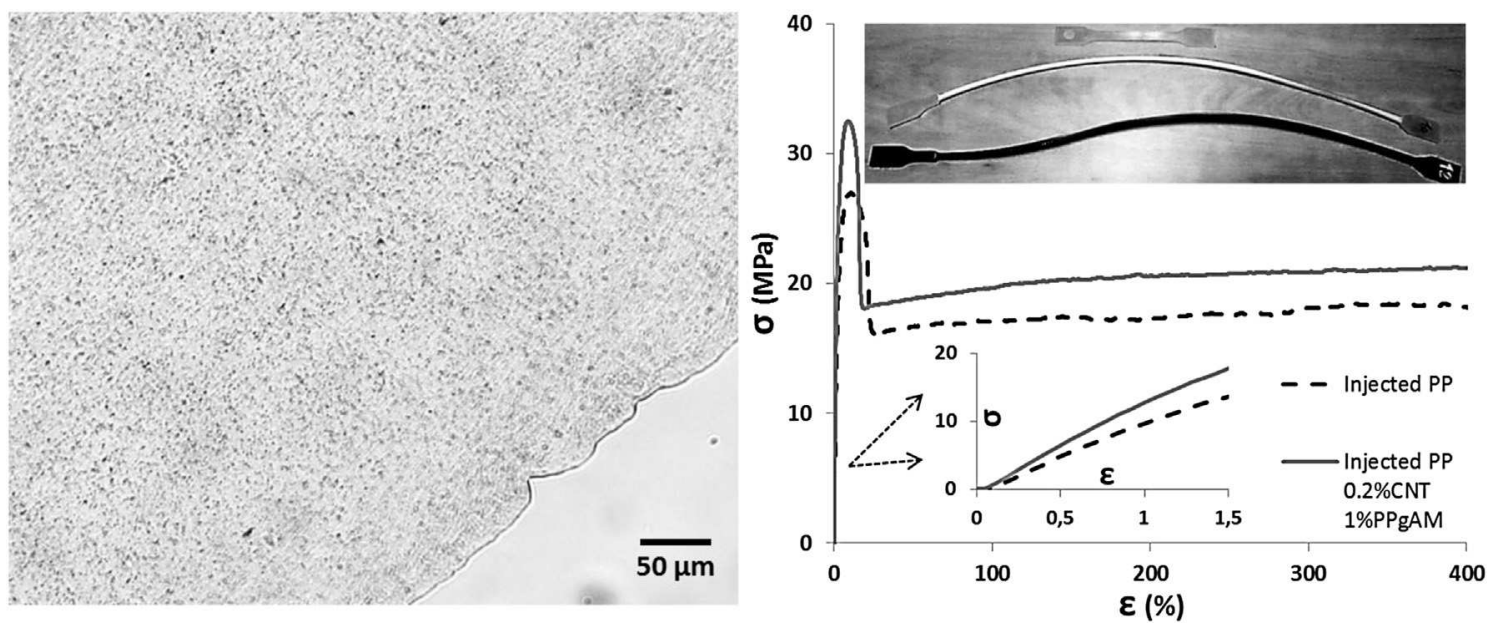

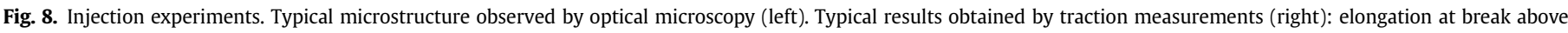
$400 \%$ (top picture and main graph), significant increase in modulus illustrated by a close-up of the traction curves in the small deformation region (inset). 
composites [40]. According to this model based on force balance and empirical data, widely used for composites, the composite modulus can be estimated as follow:

$E_{\mathrm{comp}}=E_{\mathrm{PP}} \frac{\left(1+\xi \eta v_{f}\right)}{\left(1-\eta v_{f}\right)}$ with $\xi=2\left(\frac{l}{d}\right)$ and $\eta=\frac{E_{\mathrm{CNT}}-E_{\mathrm{PP}}}{E_{\mathrm{CNT}}+\xi E_{\mathrm{PP}}}$

where $E_{\mathrm{PP}}, E_{\mathrm{CNT}}$ and $E_{\mathrm{comp}}$ are the Young's moduli for Polypropylene, Carbon Nanotubes and the composite respectively, $v_{f}$ the volume fraction of CNT in the composite, $l$ and $d$ the average length and diameter of the nanotube.

Using an estimated value of $500 \mathrm{GPa}$ for the CNT modulus (multiwall nanotubes being "softer" than single wall nanotubes with moduli around $1 \mathrm{TPa}$ ), densities of $0.905 \mathrm{~g} / \mathrm{cm}^{3}$ for the PP, $1.66 \mathrm{~g} / \mathrm{cm}^{3}$ for the CNT, average length of $1.5 \mu \mathrm{m}$ and average diameter of $9.5 \mathrm{~nm}$ as given by the suppliers, one should expect a reinforcement $\left(E_{\mathrm{comp}} / E_{\mathrm{PP}}\right)$ of about $20 \%$ according to the Halpin Tsai equation with a $0.2 \%$ wt CNT nanocomposite (around 55\% according to the simple mixing rule equation).

It appears that experimental results are very close to this estimate, which suggests that the interphase or polymer in the vicinity of the nanotubes with properties different to the bulk, plays an important role in the reinforcement as CNT are still far from being perfectly dispersed in the matrix (leading to a higher "real" size of the filler compared to the size of an individual CNT). It also remains a challenge to prepare nanocomposites leading to such results, coupled with a preserved elongation at break, at higher concentrations of CNT.

\section{Conclusion}

We have shown in this study that multilayer coextrusion can be used as an efficient and industrially relevant tool to disperse CNT in polymer matrixes such as polypropylene. A two stepprocess, with a first dilution of commercially available masterbatches via twin-screw extrusion with a high SME and a wellchosen amount of compatibilizer, followed by multilayer coextrusion, can give rise to nanocomposites with interesting properties at very low content of CNT which is a major point as far as industrial applications are concerned. We showed especially that the elongation at break can be preserved while the Young's modulus can increase by as much as $25-30 \%$ with only $0.2 \%$ CNT. Nanocomposites also displayed significantly improved thermomechanical properties over neat polypropylene. Results suggest strongly that the elongation at break is related to a relatively good dispersion of CNT, and that the presence of bigger aggregates can be almost removed using this process. DSC studies do not provide evidence of a significant change in the crystallinity of the polymer as prepared. Finally, we showed that this technique can be used to produce pellets that can be injected while maintaining the improved mechanical properties. Further studies are planned to characterize the electrical properties of these materials, and to extend it to different polymers, especially polar ones, such as polyamides.

\section{Acknowledgments}

The authors would like to thank PSA for funding this research and providing some of the materials used in this study. We also would like to thank R. Glénat, P. Soria, E. Dandeu, A. Grandmontagne and A. Dubruc for their help in the preparation and the optical and mechanical characterizations of the samples presented in this study.

\section{References}

[1] Coleman JN, Khan U, Blau WJ, Gun'ko YK. Carbon 2006;44:1624.

[2] Ajayan PM, Stephan O, Colliex C, Trauth D. Science 1994;265:1212.

[3] Spitalsky Z, Tasis D, Papagelis K, Galiotis C. Progress in Polymer Science 2010;35:357.

[4] Coleman JN, Khan U, Gun'ko YK. Advanced Materials 2006;18:689.

[5] Masuda J, Torkelson JM. Macromolecules 2008;41:5974.

[6] Brunner PJ, Clark JT, Torkelson JM, Wakabayashi K. Polymer Engineering and Science 2012;52:1555.

[7] Pujari S, et al. Journal of Polymer Science Part B-Polymer Physics 2009;47:1426.

[8] Villmow T, Potschke P, Pegel S, Haussler L, Kretzschmar B. Polymer 2008;49:3500.

[9] Kasaliwal GR, Pegel S, Goldel A, Potschke P, Heinrich G. Polymer 2010;51:2708.

[10] Villmow T, Kretzschmar B, Potschke P. Composites Science and Technology 2010;70:2045

[11] Kasaliwal GR, Goldel A, Potschke P, Heinrich G. Polymer 2011:52:1027.

[12] Schrenk WJ. U. P. 3rd ed. (USA, 1973), vol. 3773882.

[13] Xu SX, et al. Polymer 2008;49:4861.

[14] l'Abee RMA, Vissers A, Goossens JGP, Spoelstra AB, van Duin M. Analytica Chimica Acta 2009;654:11.

[15] Zumbrunnen DA, Miles KC, Liu YH. Composites Part A: Applied Science and Manufacturing 1996;27:37.

[16] Baer E, Hiltner A, Keith HD. Science 1987;235:1015.

[17] Mueller CD, Nazarenko S, Ebeling T, Schuman TL, Hiltner A, Baer E. Polymer Engineering and Science 1997;37:355.

[18] Jarus D, Hiltner A, Baer E. Polymer Engineering and Science 2001;41:2162.

[19] Jin Y, Rogunova M, Hiltner A, Baer E, Nowacki R, Galeski A, et al. Journal of Polymer Science Part B-Polymer Physics 2004;42:3380.

[20] Ponting M, Hiltner A, Baer E. Macromolecular Symposia 2010;294:19.

[21] Carr JM, Langhe DS, Ponting MT, Hiltner A, Baer E. Journal of Materials Research May, 2012;27:1326.

[22] Boufarguine M, Guinault A, Miquelard-Garnier G, Sollogoub C. Macromolecular Materials and Engineering. Article first published online: 19 Dec 2012. http://dx.doi.org/10.1002/mame.201200285.

[23] Miquelard-Garnier G, Guinault A, Sollogoub C, Delalande S, Fromonteil D. French Patent, INPI, 2012n FR1259010.

[24] Zumbrunnen DA, Inamdar S, Kwon O, Verma P. Nano Letters 2002;2:1143.

[25] Jimenez GA, Jana SC. Composites Part A: Applied Science and Manufacturing 2007;38:983.

[26] Jimenez GA, Jana SC. Carbon 2007;45:2079.

[27] Shen JB, Li J, Guo SY. Polymer Composites 2012;33:693.

[28] Wen M, Sun X, Su L, Chen J, Li J, Guo S. Polymer 2012;53:1602.

[29] Manchado MAL, Valentini L, Biagiotti J, Kenny JM. Carbon 2005;43:1499.

[30] Leelapornpisit W, Ton-That MT, Perrin-Sarazin F, Cole KC, Denault J, Simard B. Journal of Polymer Science Part B-Polymer Physics 2005;43:2445.

[31] Koval'chuk AA, Shchegolikhin AN, Shevchenko VG, Nedorezova PM, Klyamkina AN, Aladyshev AM. Macromolecules 2008;41:3149.

[32] Kovalchuk AA, Shevchenko VG, Shchegolikhin AN, Nedorezova PM, Klyamkina AN, Aladyshev AM. Journal of Materials Science 2008;43:7132

[33] Koval'chuk AA, Shevchenko VG, Shchegolikhin AN, Nedorezova PM, Klyamkina AN, Aladyshev AM. Macromolecules 2008;41:7536.

[34] Micusik M, Omastova M, Krupa I, Prokes J, Pissis P, Logakis E, et al. Journal of Applied Polymer Science 2009;113:2536.

[35] Zhao P, Wang K, Yang H, Zhang Q, Du R, Fu Q. Polymer 2007;48:5688.

[36] Muller MT, Krause B, Kretzschmar B, Potschke P. Composites Science and Technology 2011;71:1535.

[37] Perie T, Brosse AC, Tence-Girault S, Leibler L. Polymer 2012;53:984

[38] Muller MT, Krause B, Potschke P. Polymer 2012;53:3079.

[39] Amash A, Zugenmaier P. Journal of Applied Polymer Science 1997;63:1143.

[40] Halpin JC, Kardos JL. Polymer Engineering and Science 1976;16:344. 